\title{
Computation of k-out-of-n System Reliability via Reduced Ordered Binary Decision Diagrams
}

\author{
Ali Muhammad Ali Rushdi ${ }^{1}$ and Alaa Mohammad Alturki ${ }^{{ }^{*}}$
}

${ }^{1}$ King Abdulaziz University, PO.Box 80204, Jeddah 21589, Saudi Arabia.

Authors' contributions

\begin{abstract}
This work was carried out in collaboration between both authors. Author AMAR designed the study, managed the analysis, wrote the first draft of the manuscript and managed literature survey. Author AMA contributed to the analysis, implemented the algorithm, drew the figures and contributed to literature survey. Both authors read and approved the final manuscript.
\end{abstract}

Article Information

DOI: 10.9734/BJMCS/2017/33642

Editor(s):

(1) Serkan Araci, Hasan Kalyoncu University, Turkey.

Reviewers:

(1) Abdullah Sonmezoglu, Bozok University, Turkey. (2) Qingzhen Xu, South China Normal University, China.

(3) Sushil Ghimire, Kantipur Engineering College, Tribhuvan University, Nepal. Complete Peer review History: http://www.sciencedomain.org/review-history/19261

Original Research Article

Received: $24^{\text {th }}$ April 2017

Accepted: $25^{\text {th }}$ May 2017

Published: $31^{\text {st }}$ May 2017

\begin{abstract}
A prominent reliability model is that of the partially-redundant (k-out-of-n) system. We use algebraic as well as signal-flow-graph methods to explore and expose the AR algorithm for computing k-out-of-n reliability. We demonstrate that the $\mathrm{AR}$ algorithm is, in fact, both a recursive and an iterative implementation of the strategy of Reduced Ordered Binary Decision Diagrams (ROBDDs). The underlying ROBDD for the AR recursive algorithm is represented by a compact Signal Flow Graph ( $\mathrm{SFG}$ ) that is used to deduce AR iterative algorithms of quadratic temporal complexity and linear spatial complexity. Extensions of the AR algorithm for (single or scalar) threshold, double-threshold, vectorthreshold, and k-to-l-out-of-n systems have similar ROBDD interpretations.
\end{abstract}

Keywords: AR algorithm; -out-of-n system; reduced ordered binary decision diagram; reliability; signal flow graph.

\footnotetext{
*Corresponding author: E-mail: amt666@hotmail.com,amtamt666@gmail.com;
} 


\section{Introduction}

The k-out-of-n:G (F) system, is "a system of $n$ components that functions (fails) if at least $k$ out of its $n$ components function (fail)". Situations in which this system serves as a useful model are frequently encountered in practice [1-4]. The k-out-of-n system plays a central role for the general class of coherent systems, as it can be used to express or approximate the reliability of such systems with knowledge of the signatures or the destruction spectrum of such systems [4-5].

This paper deals with the evaluation of k-out-of-n system reliability via the Reduced-Ordered-BinaryDecision-Diagram (ROBDD) strategy. The ROBDD strategy was proposed by Bryant [6] as an extension of the Binary-Decision-Diagram (BDD) methodology of Akers [7]. The ROBDD deals with general switching (two-valued Boolean) functions, and is now considered the state-of-the-art data structure for handling such functions. The ROBDD has many applications in reliability theory and engineering [1,8-14]. Most of the ROBDD applications in reliability concentrate on areas which have clear-cut Boolean interpretations, such as those of fault-tree analysis or event-tree analysis [9]. In the worst case, ROBDD applications have exponential (and hence, intractable) complexity. One of a few notable exceptions is the case of applying the ROBDD to k-out-of-n systems.

The purpose of this paper is to demonstrate that simple application of the ROBDD strategy to k-out-of-n system reliability analysis produces an already existing algorithm, namely the recursive AR algorithm of Rushdi [3,15-17]. Careful implementations of the ROBDD strategy can be used to recover iterative versions of the AR algorithm.

The organization of the remainder of this paper is as follows. Section 2 reviews the AR algorithm both in the Boolean and probability domains and illustrates it via a signal-flow-graph representation. Section 3 explains why the AR algorithm is a special case of the ROBDD strategy, while Sec. 4 demonstrates the findings of Sec. 3 visually from the perspective of k-out-of-5:G systems, where $\mathrm{k}$ ranges from 1 to 5 . Section 5 concludes the paper.

\section{The AR Algorithm}

The success $\mathrm{S}\left(\mathbf{X}_{\mathrm{n}}\right)$ of a certain system in terms of the successes $\mathbf{X}_{\mathrm{n}}=\left[\mathrm{X}_{1} \mathrm{X}_{2} \ldots \mathrm{X}_{\mathrm{j}-1} \mathrm{X}_{\mathrm{j}} \mathrm{X}_{\mathrm{j}+1} \ldots \mathrm{X}_{\mathrm{n}}\right]^{\mathrm{T}}$ of its components can be expanded via the Boole-Shannon expansion w.r.t. the success $\mathrm{X}_{\mathrm{j}}$ of component $\mathrm{j}$ [17]

$$
\mathrm{S}\left(\mathbf{X}_{\mathrm{n}}\right)=\overline{\mathrm{X}}_{\mathrm{j}}\left(\mathrm{S}\left(\mathbf{X}_{\mathrm{n}}\right) / \overline{\mathrm{X}}_{\mathrm{j}}\right) \vee \mathrm{X}_{\mathrm{j}}\left(\mathrm{S}\left(\mathbf{X}_{\mathrm{n}}\right) / \mathrm{X}_{\mathrm{j}}\right), \quad 1 \leq \mathrm{j} \leq \mathrm{n}
$$

This expansion expresses $\mathrm{S}\left(\mathbf{X}_{\mathrm{n}}\right)$ in terms of two Boolean quotients (also called ratios, subfunctions or restrictions) [18-19]

$$
\begin{aligned}
& \left.\mathrm{S}\left(\mathbf{X}_{\mathrm{n}}\right) / \bar{X}_{\mathrm{j}}=\mathrm{S}\left(\mathbf{X}_{\mathrm{n}}\right)\right]_{\mathrm{Xj}=0}, \\
& \left.\mathrm{~S}\left(\mathbf{X}_{\mathrm{n}}\right) / \mathrm{X}_{\mathrm{j}}=\mathrm{S}\left(\mathbf{X}_{\mathrm{n}}\right)\right]_{\mathrm{Xj}=1 .}
\end{aligned}
$$

Rushdi $[15,17]$ proved that for the success of a k-out-of-n:G system, denoted herein as $\mathrm{S}\left(\mathrm{k}, \mathrm{n}, \mathbf{X}_{\mathrm{n}}\right)$, the above quotients (for $1 \leq \mathrm{j} \leq \mathrm{n}$ ) are

$$
\begin{aligned}
& \mathrm{S}\left(\mathrm{k}, \mathrm{n}, \mathbf{X}_{\mathrm{n}}\right) / \bar{X}_{\mathrm{j}}=\mathrm{S}\left(\mathrm{k}, \mathrm{n}-1, \mathbf{X}_{\mathrm{n}} / \mathrm{X}_{\mathrm{j}}\right) \\
& \mathrm{S}\left(\mathrm{k}, \mathrm{n}, \mathbf{X}_{\mathrm{n}}\right) / \mathrm{X}_{\mathrm{j}}=\mathrm{S}\left(\mathrm{k}-1, \mathrm{n}-1, \mathbf{X}_{\mathrm{n}} / \mathrm{X}_{\mathrm{j}}\right)
\end{aligned}
$$


where $X_{n} / X_{j}=\left[X_{1} X_{2} \ldots X_{j-1} X_{j+1} \ldots X_{n}\right]^{T}$. Note that the slash ( / ) in (1) - (5) has the meaning of restriction (or with a little abuse of notation, that of division), while in $\left(\mathbf{X}_{\mathrm{n}} / \mathrm{X}_{\mathrm{j}}\right)$ its meaning resembles that of a set difference, and hence it is used to exclude an element $X_{j}$ from an n-element vector $\mathbf{X}_{n}$ to produce an $(n-1)$ element vector $\left(\mathbf{X}_{\mathrm{n}} / \mathrm{X}_{\mathrm{j}}\right)$. When equations (1) - (5) are combined (with $\mathrm{n}$ set to $\mathrm{j}$ ), one obtains

$$
\mathrm{S}\left(\mathrm{k}, \mathrm{j}, \mathbf{X}_{\mathrm{j}}\right)=\bar{X}_{\mathrm{j}} \mathrm{S}\left(\mathrm{k}, \mathrm{j}-1, \mathbf{X}_{\mathrm{j}-1}\right) \vee \mathrm{X}_{\mathrm{j}} \mathrm{S}\left(\mathrm{k}-1, \mathrm{j}-1, \mathbf{X}_{\mathrm{j}-1}\right), \quad 1 \leq \mathrm{k} \leq \mathrm{j} \leq \mathrm{n} .
$$

The above recursive relation should be used in conjunction with the boundary conditions

$$
\begin{aligned}
& \mathrm{S}\left(0, \mathrm{j}, \mathbf{X}_{\mathrm{j}}\right)=1, \quad \mathrm{j} \geq 0 . \\
& \mathrm{S}\left(\mathrm{j}+1, \mathrm{j}, \mathbf{X}_{\mathrm{j}}\right)=0, \quad \mathrm{j} \geq 0 .
\end{aligned}
$$

Since Equations (6) and (7) are probability-ready expressions (PREs) [19], they are converted immediately to the probability domain by replacing Boolean variables by their expectations and substituting the arithmetic operations of multiplication and addition for their logical counterparts of ANDing and ORing, namely

$$
\begin{aligned}
& R\left(k, j, \mathbf{p}_{j}\right)=q_{j} R\left(k, j-1, \mathbf{p}_{j-1}\right)+p_{j} R\left(k-1, j-1, \mathbf{p}_{j-1}\right), 1 \leq k \leq j \leq n, \\
& R\left(0, j, \mathbf{p}_{j}\right)=1.0, \quad j \geq 0, \\
& R\left(j+1, j, \mathbf{p}_{j}\right)=0.0, \quad j \geq 0 .
\end{aligned}
$$

Equations (6) and (7) are the basis of the AR algorithm in the Boolean domain, while equations (8) and (9) constitute its essence in the probability domain. This algorithm is a quadratic-time iterative algorithm that has the beautiful characteristic of having a common complexity of $\mathrm{O}(\mathrm{k}(\mathrm{n}-\mathrm{k}+1))$ for computing both the unreliability and reliability of either the k-out-of-n:F system or the k-out-of-n:G system [3,19]. Figs. 1 and 2 are signal flow graphs (SFGs) that represent the underlying relations (6) and (7) for the AR algorithm in the Boolean domain, and the transformed relations (8) and (9) for that algorithm in the probability domain. The figures are drawn on a rectangular grid of a vertical coordinate $\mathrm{k}_{1}=\mathrm{k}\left(\mathrm{k}_{1} \geq 0\right)$ and a horizontal coordinate $\mathrm{k}_{2}$ $=\mathrm{j}-\mathrm{k}\left(\mathrm{k}_{2} \geq-1\right)$. Shaded circles represent nodes expressed recursively, while squares represent boundary conditions of specific values 0 and 1 for white and black nodes, respectively. Figs. 1 and 2 are useful for visualizing both the recursive and iterative versions of the AR algorithm [17]. At least three iterative versions are possible via the recursion removal attained when one traverses or sweeps the rows, columns, or secondary diagonals in Figs. 1 and 2. The arrows in Figs. 1 and 2 indicate signal-flow-graph relations, and as such, are opposite to what would be used conventionally in an expansion graph.

\section{Comparison of the AR Algorithm with the ROBDD Strategy}

The AR algorithm is, in fact, an implementation of the Reduced-Ordered-Binary-Decision-Diagram (ROBDD) strategy when this strategy is adapted for computing the k-out-n-reliability. The ROBDD deals with general switching (two-valued Boolean) functions. The AR algorithm, however, handles a class of switching functions that are both monotonically non-decreasing and totally symmetric. Apart from this restriction in applicability, the recursive version of AR algorithm has exactly the same features as the ROBDD algorithm, namely:

1. Both the AR and ROBDD algorithms are based on the Boole-Shannon expansion in the Boolean domain (1), and both use boundary conditions such as those in (7) to terminate this expansion.

2. Both algorithms visit the variables in a certain order, typically monotonically ascending or monotonically descending.

3. Both algorithms reduce the resulting expansion tree (which is exponential in size) to a rooted acyclic graph that is both canonical and hopefully compact or sub-exponential. The reduction rules [4] require (a) merging isomorphic subtrees, and (b) deletion of any useless intermediate node 
whose two "outgoing" edges point both to the same child node. For the AR algorithm, the resulting graph is not only 'hopefully' compact, but is guaranteed to correspond to quadratic temporal complexity and linear spatial complexity. Moreover, in the AR algorithm, reduction rule (b) is never needed, as these are no useless intermediate nodes. In signal-flow-graph notation, two 'outgoing' edges are, in fact, two 'incoming' transmittances. Every circled node in Figs. 1 and 2 receives transmittances from exactly two distinct nodes that do not share the same value.

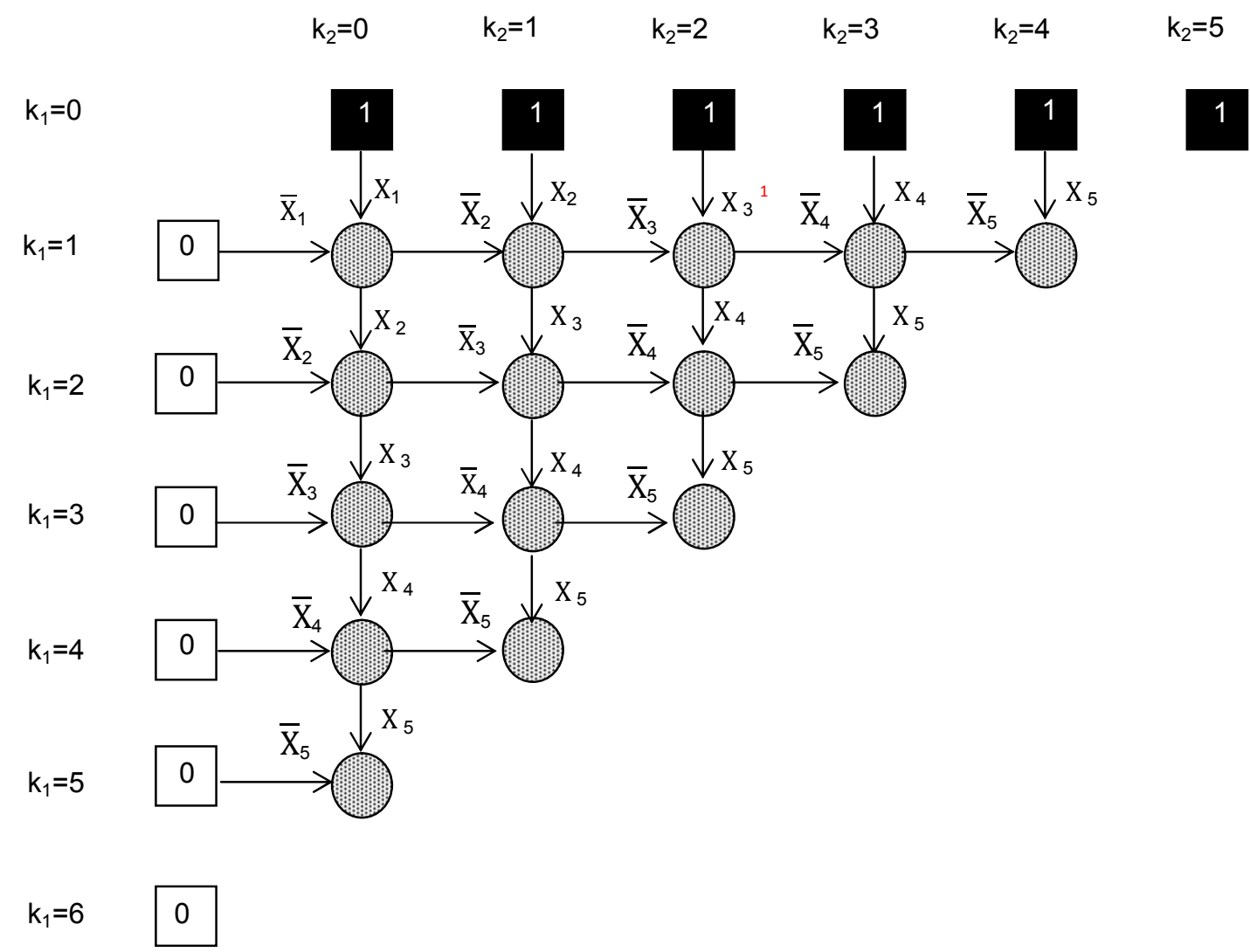

Fig. 1. The signal flow graph representing the underlying relations of the AR algorithm in the Boolean domain (operators of Boolean ANDing and ORing are assumed)

Therefore, the AR algorithm can be seen to be a subclass of ROBDD algorithms that is tailored specifically for the following equivalent purposes:

(a) Handling monotone symmetric switching functions,

(b) Computing k-out-of-n reliability; and

(c) Computing the Complementary Cumulative Distribution Function (CCDF) of the generalized Binomial Distribution.

Contrary to claims made in the literature (see, e.g., [20]), the AR algorithm is not only recursive but it has several iterative versions as well, that first appeared in [15] and reproduced in expository detail in $[3,17]$. There are ROBDD-like extensions of the AR algorithm, which handle (single or scalar) threshold systems [16,21], double-threshold systems [22], vector-threshold systems [23], and k-to-l-out-of-n systems [24]. 


\section{Demonstration via the k-out-of-5: G System}

To demonstrate that the AR algorithm is the ROBDD algorithm for computing the k-out-of-n reliability, we use Fig. 3 to depict the various ROBDDs used in the evaluation of $R(k, 5, \mathbf{p}), 1 \leq \mathrm{k} \leq 5$, which are each analogous to the related subgraphs in either Figs. 1 or 2. In an ROBDD, a node does not depict a physical entity such as a reliability value but represents a decision point and hence is labeled by the pertinent decision variable. The two emanating edges of each node are labeled by 0 and 1 values representing the two states of the decision variable. These 0 and 1 values are analogous to the $\bar{X}_{i}$ and $X_{i}$ transmittances on the SFG of Fig. 1, and also to the $\mathrm{q}_{\mathrm{i}}$ and $\mathrm{p}_{\mathrm{i}}$ transmittances on the SFG of Fig. 2.

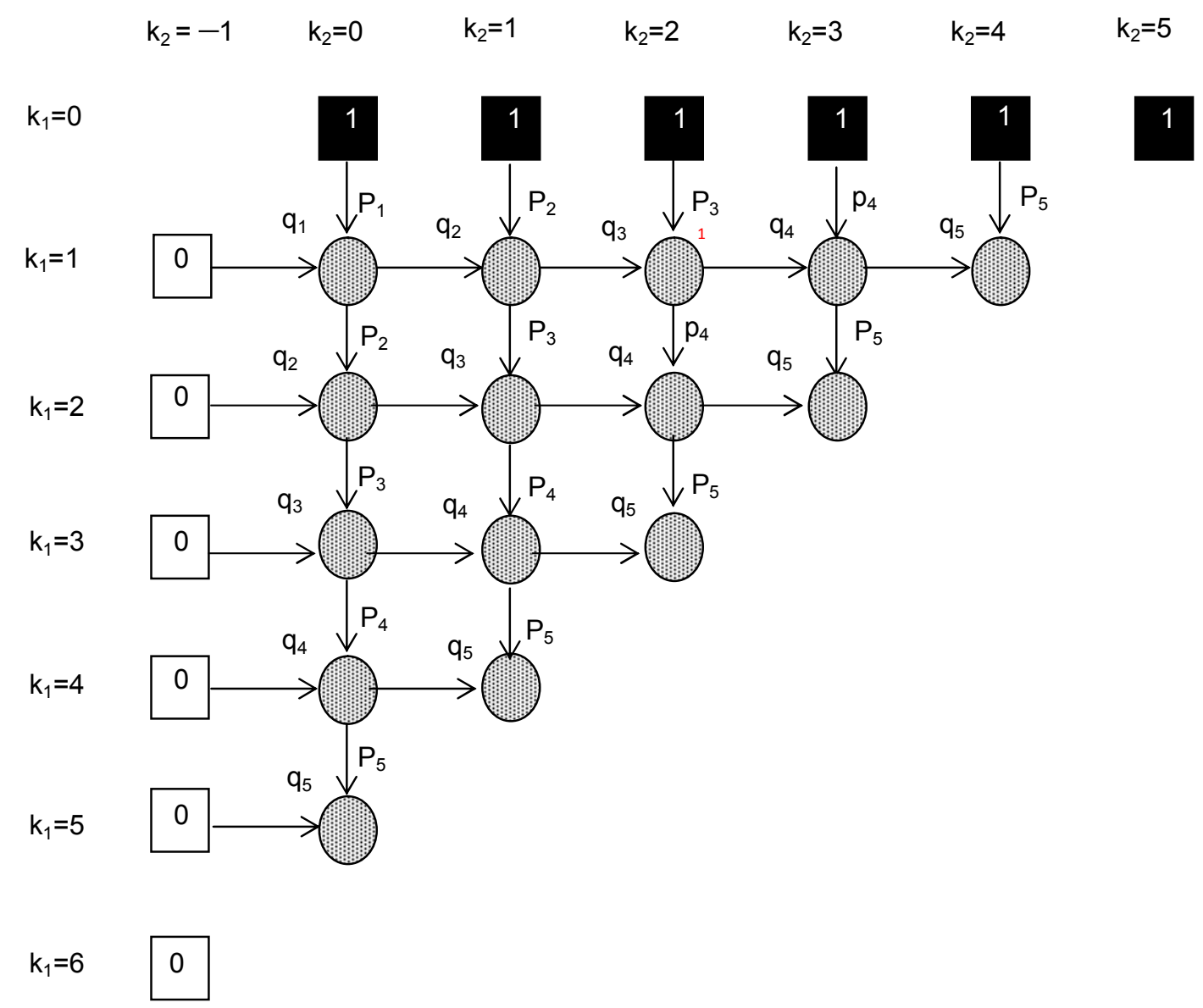

Fig. 2. The Signal Flow Graph representing the underlying relations of the AR algorithm in the probability domain (operators of arithmetic addition and multiplication are assumed)

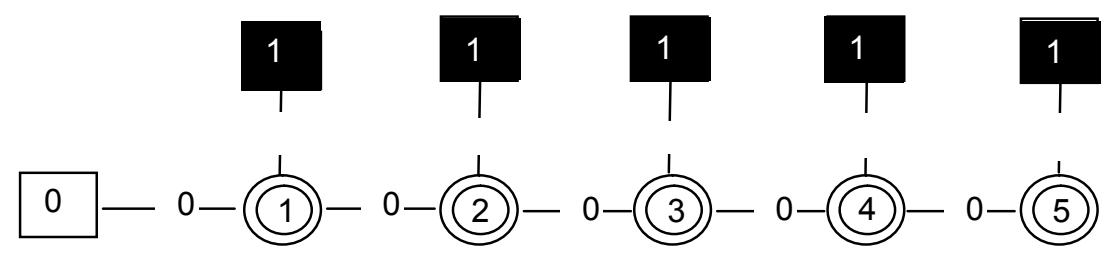

(a) 


$$
0
$$

(b)

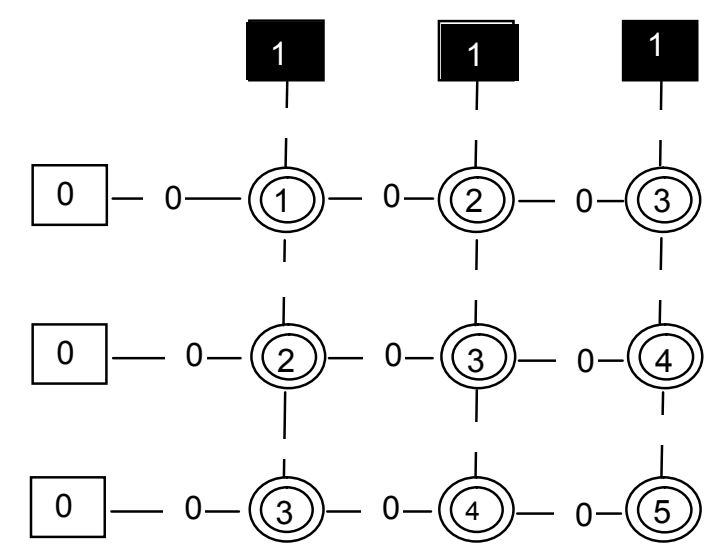

(c)

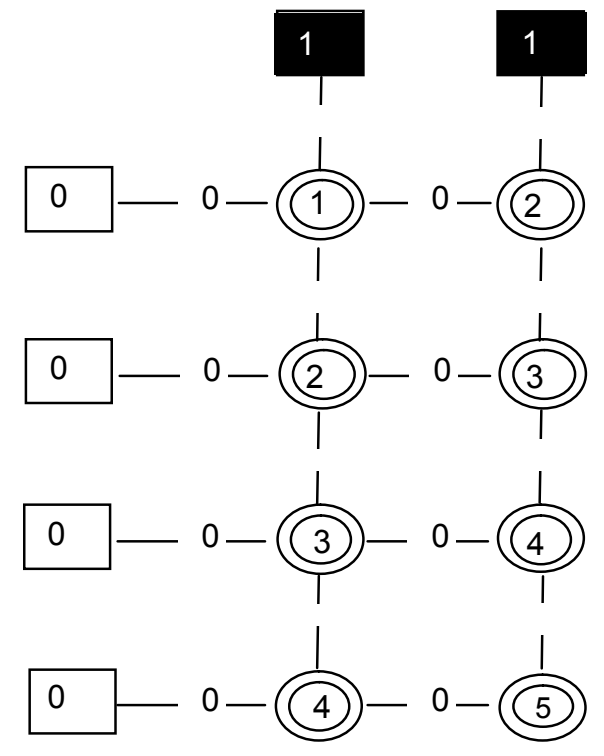

(d) 


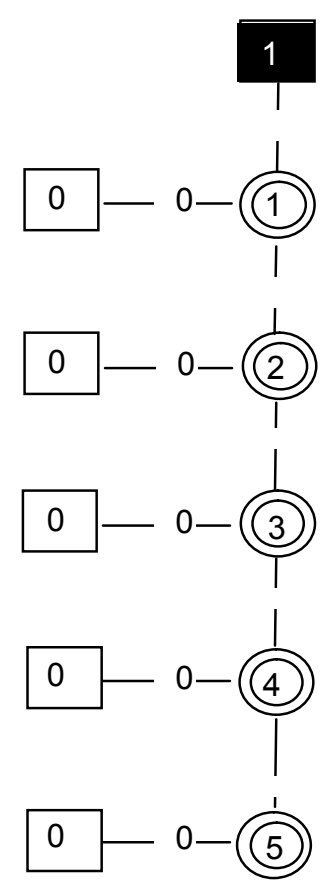

(e)

Fig. 3. The individual ROBDDs used in the computation of $R(k, 5, p), 1 \leq k \leq 5$. For simplicity, split (rather than combined) leaf nodes of 0 and 1 are used

\section{Conclusions}

We demonstrate algebraically and visually that the AR algorithm for the computing of k-out-of-n reliability is simply the particular ROBDD algorithm for such a computation. While ROBDD algorithms are generally intractable (of exponential complexity), this particular ROBDD algorithm is of quadratic temporal complexity. The results obtained herein are immediately extendible to systems generalizing the k-out-of-n system such as the (single-) threshold systems (also called weighted k-out-of-n systems), the doublethreshold systems, the vector-threshold systems and k-to-l-out-of-n systems.

\section{Competing Interests}

Authors have declared that no competing interests exist.

\section{References}

[1] Misra KB. (Editor). Handbook of performability engineering. Springer, London, UK; 2008.

[2] Amari SV, Zuo MJ, Dill GO. (kn) algorithms for analyzing repairable and non-repairable k-out-of-n: G systems. In Handbook of Performability Engineering. Springer London. 2008;309-320.

[3] Rushdi AM. Partially-redundant systems: Examples, reliability, and life expectancy. International Magazine on Advances in Computer Science and Telecommunications. 2010;1(1):1-13. 
[4] Rushdi AMA, Alturki AM. Unification of mathematical concepts and algorithms of k-out-of-n system reliability: A perspective of improved disjoint products. Journal of Engineering Research. 2017;5.

[5] Boland PJ, Samaniego FJ. The signature of a coherent system and its applications in reliability. In: Soyer, R., Mazzuchi, T. A., Singpurwalla, N. D. (eds.) Mathematical reliability: An expository perspective. Kluwer Academic Publishers, Boston, MA, USA. 2004;3-30.

[6] Bryant R. Graph-based algorithms for Boolean function manipulation. IEEE Transactions on Computers. 1986;35(8):677-691.

[7] Akers S. Binary decision diagrams. IEEE Transaction on Computers. 1960;C-27(6):509-516.

[8] Zang X, Wang D, Sun H, Trivedi KS. A BDD-based algorithm for analysis of multistate systems with multistate components. IEEE Transactions on Computers. 2003;52(12):1608-1618.

[9] Rauzy A. Binary decision diagrams for reliability studies. Chapter 25 in Misra, K. B. (Editor). Handbook of performability engineering. Springer, London, UK. 2008;381-396.

[10] Myers AF, Rauzy A. Assessment of redundant systems with imperfect coverage by means of binary decision diagrams. Reliability Engineering \& System Safety. 2008;93(7):1025-1035.

[11] Xing L. An efficient binary-decision-diagram-based approach for network reliability and sensitivity analysis. IEEE Transactions on Systems, Man, and Cybernetics-Part A: Systems and Humans. 2008;38(1):105-115.

[12] Bjorkman K. Solving dynamic flowgraph methodology models using binary decision diagrams. Reliability Engineering \& System Safety. 2013;111:206-16.

[13] Zhai Q, Peng R, Xing L, Yang J. Binary decision diagram-based reliability evaluation of k-out-of-(n$\mathrm{k}+1$ ) warm standby systems subject to fault-level coverage. Proceedings of the Institution of Mechanical Engineers, Part O: Journal of Risk and Reliability. 2013;227(5):540-548.

[14] Mo Y. A multiple-valued decision-based approach to solve dynamic fault tree. IEEE Transactions on Reliability. 2014;63(1):81-93.

[15] Rushdi AM. Utilization of symmetric switching functions in the computation of k-out-of-n system reliability. Microelectronics and Reliability. 1986;26(5):973-987.

[16] Rushdi AM. Threshold systems and their reliability. Microelectronics and Reliability. 1990;30(2): 299-312.

[17] Rushdi AM. Reliability of k-out-of-n systems. Chapter 5 in Misra, K. B. (Editor), New trends in system reliability evaluation, fundamental studies in engineering. Elsevier Science Publishers, Amsterdam, The Netherlands. 1993;16:185-227.

[18] Brown F. Boolean reasoning: The logic of Boolean equations. Kluwer Academic Publishers, Boston, USA; 1990.

[19] Rushdi AM, Rushdi MA. Switching-algebraic analysis of system reliability. Chapter 6 in Ram, M. and Davim, P. (Editors). Advances in reliability and system engineering, Management and industrial engineering series. Springer International Publishing, Cham, Switzerland. 2016;139-161.

[20] Dutuit Y, Rauzy A. New Insight into the Assessment of k-out-of-n and related systems. Reliability Engineering and System Safety. 2001;72(2):303-314. 
[21] Rushdi AM, Alturki AM. Reliability of coherent threshold systems. Journal of Applied Science. 2015; 15(3):431-443.

[22] Rushdi AMA, Bjaili HA. An ROBDD algorithm for the reliability of double-threshold systems. British Journal of Mathematics and Computer Science. 2016;19(6):1-17.

[23] Rushdi AMA, Alturki AM. An application of reliability analysis techniques in project management. British Journal of Mathematics \& Computer Science. 2017;21(6):1-15.

[24] Rushdi AM, Dehlawi F. Optimal computation of k-to-l-out-of-n system reliability. Microelectronics and Reliability. Erratum, 1988;27(5):875-896, ibid. 1987;28(4):671.

(C) 2017 Rushdi and Alturki; This is an Open Access article distributed under the terms of the Creative Commons Attribution License (http://creativecommons.org/licenses/by/4.0), which permits unrestricted use, distribution, and reproduction in any medium, provided the original work is properly cited.

\section{Peer-review history:}

The peer review history for this paper can be accessed here (Please copy paste the total link in your browser address bar)

http://sciencedomain.org/review-history/19261 